\section{Can the critically ill patient generate sufficient energy to facilitate exercise in the} ICU?

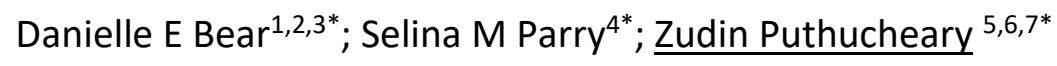

1Department of Nutrition and Dietetics, Guy's and St Thomas' NHS Foundation Trust, London, UK; 'Department of Critical Care, Guy's and St Thomas' NHS Foundation Trust, London, UK; ${ }^{3}$ Lane Fox Research Unit, Guy's and St Thomas' NHS Foundation Trust, London, UK' ${ }^{4}$ Department of Physiotherapy, The University of Melbourne, Melbourne, Victoria, Australia; ${ }^{5}$ Centre for Human Health and Performance, Department of Medicine, University College London, London, UK; ${ }^{6}$ Adult Intensive Care Unit, Royal Brompton Hospital London, UK; ${ }^{7}$ Centre of Human and Aerospace Physiological Sciences, King's College London, London, UK * All authors contributed equally

\section{Word count: 2447 /2500}

\section{Corresponding author details:}

Zudin A. Puthucheary

Zudin.puthucheary.09@ucl.ac.uk

Institute of Sport, Exercise and Health

$1^{\text {st }}$ Floor, 170 Tottenham Court Road

London W1T 7HA

United Kingdom

\section{Abstract}

Purpose of review: Trials of physical rehabilitation post critical illness have yet to deliver improved health related quality of life in critical illness survivors. Muscle mass and strength are lost rapidly in critical illness and a proportion of patients continues to do so resulting in increased mortality and functional disability. Addressing this issue is therefore fundamental for recovery from critical illness Recent findings: Altered mitochondrial function occurs in the critically ill and is likely to result in decreased Adenosine Tri-Phosphate (ATP) production. Muscle contraction is a process that requires ATP. The metabolic demands of exercise are poorly understood in the ICU setting. Recent research has highlighted that there is significant heterogeneity in energy requirements between critically ill individuals undertaking the same functional activities, such as sit-to-stand. Nutrition in the critically ill is currently thought of in terms of carbohydrates, fat and protein. It may be that we need to consider nutrition in a more contextual manner such as energy generation or management of protein homeostasis.

Summary: Current nutritional support practices in critically ill patients do not lead to improvements in physical and functional outcomes, and it may be that alternative methods of delivery or substrates are needed.

Key words: critically ill, muscle wasting, exercise, nutrition 


\section{Introduction}

Physical rehabilitation trials have yet to deliver increased functional capacity or improve health related quality of life in critical illness survivors ${ }^{1}$. All things being equal, muscle mass is directly related to muscle strength, and is therefore a major determinant of physical function. Regardless of the measurement method used, muscle mass and strength is lost rapidly in critical illness ${ }^{2}$, a process that continues post critical illness ${ }^{3}$. This is associated with increased mortality ${ }^{4}$ and functional disability $^{3}$. Addressing this issue is therefore fundamental for recovery from critical illness. Muscle mass is maintained by protein homeostasis, a highly energy dependent process. Without sufficient energy generation, exercise interventions will not succeed in maintenance of protein homeostasis.

\section{The bioenergetics status of the critically ill patient}

Altered mitochondrial function occurs in sepsis and multi-organ failure ${ }^{5}$ that is likely to result in decreased Adenosine Tri-Phosphate (ATP) production. The causality of this observed phenomena is unclear, altered mitochondrial function being either: a response to extra-cellular stimuli (e.g. inflammation), or a protective mechanism (to prevent utilization of biological stores). For the patient, the resulting phenotype is the same. Lack of ATP will hinder muscle protein synthesis (MPS), with acute muscle wasting leading to functional disability on discharge. Further lack of ATP may trigger proteolysis, accelerating loss of muscle mass ${ }^{6}$. Altered mitochondrial function may additionally affect muscle satellite cells ${ }^{7}$ altering regenerative capacity, which in turn would lead to altered muscle quality ${ }^{8}$ further impairing muscle function.

ATP is generated by the linking of oxidation of substrate to phosphorylation of Adenosine Di-Phosphate, i.e. oxidative phosphorylation. ATP is additionally necessary for skeletal muscle contraction, leading to force generation i.e. exercise. Interestingly this is a bidirectional relationship: exercise training is able to increase the capacity for ATP supply and mitochondrial density ${ }^{9}$. Glycolysis produces pyruvate for the Citric Acid cycle and high-energy electrons for the electron transport chain. Fatty Acid Oxidation produces Nicotinamide adenine dinucleotide (NADH) and Flavin adenine dinucleotide $\left(\mathrm{FADH}_{2}\right)$ and Acetyl-Coenzyme-A, which are used in the Citric Acid Cycle and the electron transport chain ${ }^{10}$. These are enzyme driven processes- in the setting of altered mitochondrial function, increasing substrate delivery is unlikely to alter substrate processing, and therefore unlikely to produce more ATP.

Two processes common to critical illness may alter pre-intensive care unit (ICU) mitochondrial function and subsequent ATP generation- age and chronic disease states. Aging muscle is associated with decreased mitochondrial efficiency, though there has been little control for sedentary behavior. Immobilization does result in decreased mitochondrial efficiency. This may contribute to the altered mitochondrial function seen with chronic disease states either at rest or following a physiological stressor. This complex metabolic state is likely to be a result of metabolic adaptation, from decreased insulin sensitivity in diabetic patients to muscle fibre type shifts in those chronic diseases associated with sedentary behavior and immobilization. In summary, not only is energy production likely to be impaired in critical illness, patients are likely to enter critical illness with varying abilities to oxidise substrate and produce ATP. 


\section{Skeletal muscle adaption to loading and energy requirements}

Myofibrils are the smallest structural unit of a muscle fibre and contain two main contractile filaments, actin (thin filaments) and myosin (thick filaments), which are responsible for contractile functioning and force production. The interaction of these filaments induces muscle contraction - a process that requires ATP. Muscle fibres exhibit different contraction properties based on differences in energy hydrolysis and synthesis. The terms 'slow' or 'fast' twitch fibre typing is used to describe the speed of contraction, level of microvascular capillarisation, oxidative enzymes and metabolic means of generating energy production (aerobic or anaerobic) ${ }^{11}$. The musculoskeletal system is a highly plastic and adaptive system responding quickly to changes in mechanical loading particularly due to immobility and sepsis ${ }^{12}$.

It is recognized that other factors influence muscle force production including muscle fibre arrangement, tendon stiffness, and motor unit discharge rates and neurohormonal states. Where there is reduced mechanical loading and/or increased energy demands due to heightened states of physical stress -such as seen in critical illness - imbalance in muscle protein homeostasis can ensue ${ }^{14}$.

\section{Effect of pre-ICU health and post ICU recovery on skeletal muscle dysfunction}

As with mitochondrial function (detailed above) pre-ICU health factors such as age, comorbidities and frailty influence muscle mass on admission to the ICU and the potential recovery trajectory ${ }^{13}$. Considerable patient heterogeneity results in differing responses between individuals post critical illness ${ }^{14}$, demonstrated in secondary analyses of 'negative' rehabilitation RCTs ${ }^{15}{ }^{16}$.

The mechanistic changes for muscle dysfunction in critical illness continue to be elucidated. It is established that muscle wasting occurs early and rapidly with up to $30 \%$ reduction in first 10 days ${ }^{12} 14$. Heightened muscle protein catabolism is evident in individuals with higher severity of illness ${ }^{612}$. Additionally macrophagic infiltration, myonecrosis, preferential reduction of myosin and reduction in mitochondrial content have been demonstrated in the early phases of muscle degradation 612 . Reduced motor nerve excitability with impairment in membrane excitability occurs, impacting muscle contraction ${ }^{17}$. Mechanisms of chronic muscle wasting and dysfunction six months post discharge are not just due to ongoing low muscle mass but also reduced satellite cells, angiogenesis, and increased intramuscular fat and connective tissue infiltration ${ }^{6}$, impacting on the ability of skeletal muscle to adapt and respond to changes in mechanical loading through exercise ${ }^{6}$.

\section{How can we 'load' or train skeletal muscle - Exercise Principles}

The American College of Sports Medicine (ACSM) has defined resistance training as a form of physical activity that is designed to improve muscular fitness by exercising a muscle in isolation or a muscle group against external resistance ${ }^{18}$. Training methods will vary depending on the purpose for resistance training - strength, endurance, or power ${ }^{18}$. In order to maintain muscle mass or induce hypertrophy overloading of the muscle is required. This is dependent on intact structural, neurohormonal, cardiovascular and metabolic energy system, which can be compromised in critically ill. Consideration of frequency, load resistance, type of 
exercises and volume (number of repetitions and sets) are critical to designing an efficacious training program ${ }^{18}$. To our knowledge there are no studies which have specifically examined resistance training on its own in the ICU setting - recent RCTs have incorporated resistance training as one facet within physical rehabilitation ${ }^{19} 20$.

\section{Resistance training in the ICU setting and metabolic costs}

Resistance exercises can involve functional multi-joint strengthening exercises such as sit-to-stand, which are highly important to everyday activities of daily living. The ICU environment is fraught with complexities that impact on the ability of patients to engage in physical activity. The barriers to physical activity were recently highlighted in a systematic review and include patient, clinician and institutional level influences ${ }^{21}$. With introduction of guidelines and frameworks for optimizing pain, agitation and delirium management there has been a paradigm shift towards creating an animated ICU in which patient engagement can be optimized ${ }^{22}{ }^{23}$. There still remains challenges however in the initial ICU admission period where patients may be unable to volitionally participate in rehabilitation strategies. Given muscle loss occurs early and rapidly there is a need to consider ways in which 'loading' forces may be applied to maintain muscle mass integrity.

160 The metabolic demands of exercise are poorly understood in the ICU setting. Recent 161 research has highlighted that there is significant heterogeneity in energy requirements between critically ill individuals undertaking the same functional activities $^{24}$. Energy requirements are higher in the critically ill compared to healthy individuals ${ }^{24}$. A strong correlation exists between active energy expenditure measured using a physical activity device and the highest level of mobility ${ }^{25}$. However the ability to detect low levels of activity is challenging when patients are relatively immobile compounded by potential inaccuracies in energy assessment with physical activity devices ${ }^{26}$. Additionally metabolic demands are likely to differ for in bed versus out of bed activity - but have not yet been examined.

The metabolic demand of resistance training has been examined in non-ICU settings. Eccentric muscle contraction (involving generation of muscle force during muscle lengthening) induces lower metabolic demands compared to concentric muscle contractions ${ }^{27}$. Resistance training methodology is therefore as important as considerations of the potential adverse effects of overtraining. Response to training is variable and further research into individualizing interventions whilst considering metabolic reserve and baseline and exercise energy expenditure

Future directions for the field include understanding the synergy and interaction between nutrition and exercise ${ }^{28}$. Consideration of timing of nutrition may therefore be important not only prior /during resistance training but also in the post exercise period.

\section{Substrate delivery and energy generation-not the same thing}

In health, protein, fat and carbohydrate are all required for ATP synthesis. In addition, co-factors, such as b-vitamins, and insulin are required for this process to occur. In athletes, appropriate nutrition is essential to achieving and maintaining peak exercise performance and is prioritized. Inadequate energy intake compared 
reduced muscle mass and overall strength. These same considerations may be true for critically ill patients.

Several factors require consideration regarding the role of nutrition in the generation of energy for exercise in critically ill patients. The first is adequate substrate delivery. It is well documented that critically ill patients do not receive prescribed amounts of nutrition regardless of route. Reasons for this include fasting for surgical and bedside procedures as well as high gastric residual volumes that are used as a crude bedside indication of enteral feeding intolerance. In the context of mitochondrial dysfunction described earlier, increasing substrate delivery may not improve ATP generation. Secondly, the contribution of endogenous glucose and amino acid production from the metabolic effects of stress on the overall energy needs of the patient during early critical illness is unknown. Lastly Insulin is a requirement for ATP generation. Insulin resistance during the acute phase of critical illness may therefore contribute to impaired ATP generation- the timing of nutrient provision for energy generation is likely a key factor.

Delivery of nutrition does not necessarily equate to absorption, particularly in the early course of illness. Data have shown that there are alterations in both the rate and extent of nutrient absorption from the Gl tract, even with post-pyloric feeding is utilized ${ }^{29}{ }^{30}$. This may limit the potential for nutrients to facilitate energy generation and with no quick bedside measure available to determine this elucidation of the relative contribution of poor absorption to subsequent functional disability remains unknown. Either way, delivery of nutrition should not be assumed to contribute to energy generation throughout the course of critical illness and physiological studies are required to understand this process.

\section{Can we use more efficient substrates?}

Current evidence suggests that physical rehabilitation does not lead to improvements in outcome in critically ill patients ${ }^{31}{ }^{32}$.No data exists to suggest current nutritional support practices lead to improvements in rehabilitation potential and physical and functional outcomes, as unfortunately the nutritional status and intake of the patients has not been reported in such trials.

Nutrition in the ICU is currently thought of in terms of carbohydrates, fat and protein. Recently, particular attention has been given to protein with updated guidelines recommending higher amounts in order to prevent loss of lean body mass $^{33}$. Physiological studies have shown that protein intakes equivalent to $1 \mathrm{~g} / \mathrm{kg} /$ day increase whole body protein turnover, thought to be as a result of increased protein synthesis. One small study has shown that increased delivery of amino acids to patients requiring PN increased handgrip strength at day 7 of ICU admission ${ }^{34}$ However, no studies have investigated the effect of different protein doses directly on MPS. Indeed, studies have indicated that higher protein intakes are associated with increased rates of muscle wasting ${ }^{12}$ and others have shown that weakness was increased in patients receiving higher macronutrient doses from the addition of supplemental PN. For this reason, prospective studies investigating the effect of protein intake on MPS in critical illness are urgently required ${ }^{35}$.

Exercise and nutrition go hand in hand and the nutrient or substrate requirement will be dependent on the duration and intensity of the exercise being performed. For 
example, protein intake during or immediately after resistance training has been found to increase MPS. However, continuous feeding, as in the case of the critically ill patient, make negate this physiological process due to the 'muscle full effect' whereby saturation of the muscle with amino acids does not lead to further increases in MPS. In this regard, intermittent feeding, coupled with resistance training, may improve protein turnover in critically ill patients ${ }^{29}$.

For the reasons specified above, clinicians and researchers are seeking alternative substrates to improve mitochondrial function, MPS and subsequently the strength and rehabilitation potential of these patients. Critically ill patients have been likened to athletes with an intense training (critical care admission) and recovery phase. Ergogenic supplements and a varied substrate schedule used by athletes may prove efficacious in this patient cohort and provide an alternative or additional substrate to carbohydrate, fat and protein. Such supplements may include $\beta$-hydroxy- $\beta$ methylbutyrate (HMB), Leucine, creatine, carnitine, nitrates and beta-alanine (Table 1) ${ }^{36}$. Although the exact mechanisms underlying the effect of these interventions on exercise performance remain unknown, studies in healthy and limited clinical populations are promising, albeit not consistent.

Of the above-mentioned substrates, Leucine, $\mathrm{HMB}$ and creatine are perhaps the most widely studied ${ }^{36}$. Leucine itself has been widely studied for its effects on protein synthesis, mitochondrial function, glucose homeostasis, insulin action and subsequently recovery from exercise and other catabolic conditions ${ }^{36}$. The conversion of leucine to $\mathrm{HMB}$ has been considered a key pathway in protein homeostasis ${ }^{37}$. $\mathrm{HMB}$ has been shown to attenuate the Ubiquitin Protesome Pathway (UPP) along with other catabolic pathways In addition, HMB has been shown to influence mitochondrial dynamics disuse (bed rest) and rehabilitation. Lastly, creatine has been shown to contribute to ATP synthesis during high-energy demands. Intramuscular creatine is phosphorylated to phosphocreatine which is followed by the phosphate from phosphocreatine plus free ADP being used for this process $^{38}$.

Along with these substrates, discussions around the benefit of inducing ketosis as an alternative fuel source for muscle are increasing. In early critical illness, traditional fuels may be bioenergetically inert, meaning that they are unable to contribute to ATP synthesis. Switching the fuel source to ketones may therefore be useful in this early phase to spare muscle and facilitate generation of ATP.

\section{Conclusions}

Patients may not be able to generate enough energy to undertake exercise in early critical illness due to mitochondrial dysfunction preventing ATP production. It is unlikely that one strategy alone will be successful in modifying this, but nutrition and exercise are most likely to have essential synergistic roles to play.

Key points: 
- The compromised bioenergetic status of the critically ill patient is unlikely to respond to increased substrate delivery

- patients are likely to enter critical illness with varying abilities to oxidize substrate and produce ATP

- The metabolic cost of resistance exercise in the critically ill patient is unknown

- delivery of nutrition should not be assumed to contribute to energy generation

- Research into alternative substrates in the critically ill is urgently needed if we are to prevent acute muscle wasting

\section{Acknowledgements}

None

Financial Support and sponsorship:

SM Parry is supported by a National Health and Medical Research Council Early Career Fellowship (\#1111640) and was a recipient of a short term European Respiratory Society Fellowship in 2016

\section{Conflicts of interest}

ZP has received honoraria for consultancy from GlaxoSmithKleine, Lyric Pharmaceuticals and Fresenius-Kabi. DEB has received speaker fees and conference attendance support from Nutricia, Baxter, BBraun, Nestle Nutrition and Fresenius Kabi; Consultancy fees through her institution from Abbott Nutrition and grant support through her institution from Corpak MedSystems UK.

\section{REFERENCES}

1. Hodgson C, Cuthbertson BH. Improving outcomes after critical illness: harder than we thought! Intensive care medicine 2016;42(11):1772-74.

2. Puthucheary ZA, McNelly AS, Rawal J, et al. Rectus Femoris Cross-Sectional Area and Muscle Layer Thickness: Comparative Markers of Muscle Wasting and Weakness. American journal of respiratory and critical care medicine 2017;195(1):136-38.

3. Pfoh ER, Wozniak AW, Colantuoni E, et al. Physical declines occurring after hospital discharge in ARDS survivors: a 5-year longitudinal study. Intensive care medicine 2016;42(10):1557-66.

4. Puthucheary Z, Prescott H. Skeletal Muscle Weakness Is Associated With Both Early and Late Mortality After Acute Respiratory Distress Syndrome. Critical care medicine 2017;45(3):563-65.

5. Arulkumaran N, Deutschman CS, Pinsky MR, et al. Mitochondrial Function in Sepsis. Shock (Augusta, Ga 2016;45(3):271-81.

6. **Batt J, Herridge M, Dos Santos C. Mechanism of ICU-acquired weakness: skeletal muscle loss in critical illness. Intensive care medicine 2017. Narrative review article summarizing the current understanding of the mechanisms behind skeletal muscle loss in critical illness 
7. Chatre L, Verdonk F, Rocheteau P, et al. A novel paradigm links mitochondrial dysfunction with muscle stem cell impairment in sepsis. Biochimica et biophysica acta 2017.

8. Dos Santos C, Hussain SN, Mathur S, et al. Mechanisms of Chronic Muscle Wasting and Dysfunction After an Intensive Care Unit Stay: A Pilot Study. American journal of respiratory and critical care medicine 2016.

9. Conley KE. Mitochondria to motion: optimizing oxidative phosphorylation to improve exercise performance. J Exp Biol 2016;219(Pt 2):243-9.

10. O'Neill LA, Kishton RJ, Rathmell J. A guide to immunometabolism for immunologists. Nat Rev Immunol 2016;16(9):553-65.

11. Ceco E, Weinberg S, Chandel N, et al. Metabolism and skeletal muscle homeostasis in lung disease. American Journal of Respiratory Cell and Molecular Biology 2017;57(1):28-34.

12. Puthucheary ZA, Rawal J, McPhail M, et al. Acute skeletal muscle wasting in critical illness. JAMA 2013;310(15):1591-600.

13. Ferrante L, Pisani M, Murphy T, et al. Factors associated with functional recovery among older intensive care unit survivors. Am J Respir Crit Care Med 2016;194:299-307.

14. ${ }^{* *}$ Latronico N, Herridge M, Hopkins RO, et al. The ICM research agenda on intensive care unit-acquired weakness. Intensive care medicine 2017. International research agenda on intensive care unit acquired weakness including discussion of what is currently known and identification of future areas of research.

15. Moss M, Nordon-Craft A, Malone D, et al. A Randomized Trial of an Intensive Physical Therapy Program for Patients with Acute Respiratory Failure. AJRCCM 2016;193(10):1101-10.

16. Neumeier A, Nordon-Craft A, Malone D, et al. Prolonged acute care and postacute care admission and recovery of physical function in survivors of acute respiratory failure: a secondary analysis of a randomized controlled trial. Critical Care 2017;21(190).

17. Koch S, Bierbrauer J, Haas K, et al. Critical illness polyneuropathy in ICU patients is related to reduced motor nerve excitability caused by reduced sodium permeability. Intensive Care Medicine Experimental 2016;4(10).

18. American College of Sports Medicine. ACSM's Guidelines for Exercise Testing and Prescription. Philadelphia: Wolters Kluwer / Lippincott Williams \& Wilkins, 2014.

19. Hodgson C, Tipping C. Physiotherapy management of intensive care unitacquired weakness. Journal of Physiotherapy 2017;63:4-10.

20. Tipping C, Harrold M, Holland A, et al. The effects of active mobilisation and rehabilitation in ICU on mortality and function: a systematic review. Intensive Care Medicine 2017;43:171-83.

21. *Parry SM, Knight LD, Connolly B, et al. Factors influencing physical activity and rehabilitation in survivors of critical illness: a systematic review of quantitative and qualitative studies. Intensive care medicine 2017;43(4):531-42. Comprehensive systematic review detailing the factors that influence physical activity and rehabilitation in survivors of critical illness. It also highlights potential modifiable barriers and enablers to increase engagement in physical activity and rehabilitation from patient, clinician and institutional level 
22. Girard T, Alhazzani W, Kress J, et al. An Official American Thoracic Society / American College of Chest Physicians Clinical Practice Guideline: Liberation from Mechanical ventilation in critically ill adults. Rehabilitation protocols, ventilator liberation protocols and cuff leak tests. American Journal of Respiratory \& Critical Care Medicine 2017;195(1):120-33.

23. Parry S, Nydahl P, Needham D. Implementing early physical rehabilitation and mobilisation in the ICU: institutional, clinician and patient considerations. intensive Care Medicine 2017;Epub Ahead of Print.

24. Black C, Singer M, Grocott M. The oxygen cost of rehabilitation in mechanically ventilated patients. American Journal of Respiratory \& Critical Care Medicine 2017;195(A2742).

25. Beach L, Fetterplace K, Edbrooke L, et al. Measurement of physical activity levels in the intensive care unit and functional outcomes: An observational study. Journal of critical care 2017;40:189-96.

26. Kruger J, Kraft M, Grundling M, et al. Evaluation of a non-invasive multisensor accelerometer for calculating energy expenditure in ventilated intensive care patients compared to indirect calorimetry and predictive equations. Journal of Clinical Monitoring and Computing 2017;31(5):1009-17.

27. Mitchell K, Taivassalo T, Narici M, et al. Eccentric exercise and the critically ill patient. Frontiers in Physiology 2017;8(120).

28.** Heyland D, Stapleton R, Mourtazkis M, et al. Combining nutrition and exercise to optimize survival and recovery from critical illness: Conceptual and methodological issues. Clinical Nutrition 2016;35(5):1196-206. Opinion perspective on conceptual and methodological issues for combining nutrition and exercise in individuals with critical illness. A common evaluation framework and methods to standardize assessments in the field is also discussed

29. **Arabi YM, Casaer MP, Chapman M, et al. The intensive care medicine research agenda in nutrition and metabolism. Intensive care medicine 2017. International research agenda on nutrition and metabolism including discussion of what is currently known and identification of future areas of research.

30. Santacruz CA, Quintairos A, Righy C, et al. Is There a Role for Enterohormones in the Gastroparesis of Critically Ill Patients? Critical care medicine 2017;45(10):1696-701.

31. Moss M, Nordon-Craft A, Malone D, et al. A Randomized Trial of an Intensive Physical Therapy Program for Patients with Acute Respiratory Failure. American journal of respiratory and critical care medicine 2016;193(10):1101-10.

32. McDowell K, O'Neill B, Blackwood B, et al. Effectiveness of an exercise programme on physical function in patients discharged from hospital following critical illness: a randomised controlled trial (the REVIVE trial). Thorax 2017;72(7):594-95.

33. McClave SA, Taylor BE, Martindale RG, et al. Guidelines for the Provision and Assessment of Nutrition Support Therapy in the Adult Critically Ill Patient: Society of Critical Care Medicine (SCCM) and American Society for Parenteral and Enteral Nutrition (A.S.P.E.N.). Jpen 2016;40(2):159211. 
34. Ferrie S, Allman-Farinelli M, Daley M, et al. Protein Requirements in the Critically Ill: A Randomized Controlled Trial Using Parenteral Nutrition. Jpen 2016;40(6):795-805.

35. *Bear DE, Wandrag L, Merriweather JL, et al. The role of nutritional support in the physical and functional recovery of critically ill patients: a narrative review. Critical care (London, England) 2017;21(1):226. Narrative review of the role of nutrition support in the physical and functional recovery of ICU survivors. Limitations to current research and considerations for future studies are discussed.

36. *Deane CS, Wilkinson DJ, Phillips BE, et al. "Nutraceuticals" in relation to human skeletal muscle and exercise. Am J Physiol Endocrinol Metab 2017;312(4):E282-E99. Comprehensive review article detailing the role of neutraceuticals in muscle metabolism and exercise performance. Their role in the presence and absence of exercise is discussed.

37. Standley RA, Distefano G, Pereira SL, et al. Effects of beta-hydroxy-betamethylbutyrate (HMB) on skeletal muscle mitochondrial content and dynamics, and lipids after 10 days of bed rest in older adults. J Appl Physiol (1985) 2017:jap 001922017.

38. He X, Duan Y, Yao K, et al. beta-Hydroxy-beta-methylbutyrate, mitochondrial biogenesis, and skeletal muscle health. Amino Acids 2016;48(3):653-64. 
454 Table 1: Potential substrates which may enhance muscle mass and exercise 455 performance in the critically ill

\begin{tabular}{|c|c|}
\hline Substrate & Current evidence \\
\hline $\begin{array}{l}\beta \text {-hydroxy- } \beta \text { - } \\
\text { methylbutyrate } \\
(\mathrm{HMB})\end{array}$ & $\begin{array}{l}\text { - Increases MPS and decreases MPB } \\
\text { - Increases lean body mass, aerobic and strength performance } \\
\text { - } \quad \text { Preserves muscle mass during bed rest, in the absence of } \\
\text { exercise }\end{array}$ \\
\hline Leucine & $\begin{array}{ll}\text { - } & \text { Increases MPS via mTOR } \\
\text { - } & \text { May increase protein uptake into muscle }\end{array}$ \\
\hline Creatine & $\begin{array}{l}\text { - Phosphate from phosphocreatine plus free ADP used for ATP } \\
\text { synthesis and therefore muscle contraction } \\
\text { - Improves rate of recovery, thereby increasing MPS }\end{array}$ \\
\hline Carnitine & $\begin{array}{l}\text { - May delay fatigue during prolonged aerobic exercise } \\
\text { - Enhances fat oxidation whilst sparing glycogen }\end{array}$ \\
\hline$\beta$-alanine & $\begin{array}{l}\text { - Increases muscle carnosine which may enhance acute exercise } \\
\text { performance }\end{array}$ \\
\hline Nitrates & $\begin{array}{l}\text { Increase blood flow and subsequently nutrient delivery to the } \\
\text { muscle leading to anabolism }\end{array}$ \\
\hline Ketones & - Muscle sparing and provide an alternative fuel source \\
\hline
\end{tabular}

